\title{
The role of central and peripheral vision in perceiving the direction of self-motion
}

\author{
WILLIAM H. WARREN and KENNETH J. KURTZ \\ Brown University, Providence, Rhode Island
}

\begin{abstract}
Three experiments were performed to examine the role that central and peripheral vision play in the perception of the direction of translational self-motion, or heading, from optical flow. When the focus of radial outflow was in central vision, heading accuracy was slightly higher with central circular displays $\left(10^{\circ}-25^{\circ}\right.$ diameter) than with peripheral annular displays (40 diameter), indicating that central vision is somewhat more sensitive to this information. Performance dropped rapidly as the eccentricity of the focus of outflow increased, indicating that the periphery does not accurately extract radial flow patterns. Together with recent research on vection and postural adjustments, these results contradict the peripheral dominance hypothesis that peripheral vision is specialized for perception of self-motion. We propose a functional sensitivity hypothesis-tha: self-motion is perceived on the basis of optical information rather than the retinal locus of stimulation, but that central and peripheral vision are differentially sensitive to the information characteristic of each retinal region.
\end{abstract}

Research on the role of vision in the perception of selfmotion has emphasized three phenomena induced by optical flow stimulation: (1) vection, or the subjective experience of self-motion; (2) postural adjustments such as body sway or tilt during standing; and (3) the perception of heading, or the direction of self-motion. To some extent, these phenomena are independent of one another, for vection and heading are commonly perceived without postural adjustments, heading can be perceived without the sensation of vection, and postural compensation has been reported with optical velocities too low to induce vection (Delorme \& Martin, 1986; Lee \& Lishman, 1975; Stoffregen, 1986). However, to the extent that vection is experienced in a definite direction and postural adjustments are directionally specific, the extraction of information about heading is implicated.

On the basis of evidence from the first two domains, claims have frequently been made for a peripheral dominance hypothesis-specifically, that peripheral vision plays the dominant role in the perception and control of selfmotion and that central vision is relatively insensitive to such information. In their review, Dichgans and Brandt (1978) concluded that, "The peripheral retina dominates visually induced vection and spatial orientation, whereas central vision dominates pattern perception and object motion detection" (p. 777; italics in original). Our purpose in the present paper is to consider the role of different retinal regions in perceiving self-motion and to include a review

This research was supported by Grant AG05223 from the National Institutes of Health. We thank Arshavir Blackwell for his work on Experiment 2, and Jim Crowell and three reviewers for their helpful comments. Correspondence should be addressed to William $H$. Warren, Department of Cognitive and Linguistic Sciences, Brown University, Providence, RI 02912. of the evidence on the peripheral dominance hypothesis and a test of it in the third domain of perceiving heading.

The central problem in perceiving self-motion is to distinguish the patterns of optical flow that are due to selfmotion from those that are due to object motion, so that they can be responded to differentially. A priori considerations suggest several possible optical bases for the perception of self-motion. First, as Gibson (1950, 1954, 1968) pointed out, movement of the observer tends to generate a global transformation of the optic array, whereas object motion tends to produce a local transformation in a bounded region of the array. (Exceptions include self-motion in a vehicle with small windows, and motion of large nearby objects.) Although there is considerable evidence that larger areas of stimulation induce stronger vection and greater postural sway, small regions of stimulation ( $<30^{\circ}$ in diameter) can still be effective. This indicates that the area of stimulation by itself cannot provide the full story.

Peripheral dominance, first proposed by Brandt, Dichgans, and Koenig (1973), could derive from similar considerations. Because self-motion tends to produce a global transformation, it is more likely to yield optical flow in the visual periphery than is local object motion, and thus the peripheral retina could have become specialized for detecting self-motion. An obvious problem is that a moving object in peripheral vision, or pursuit tracking of a moving object against a background, would also produce optical motion in the periphery. This suggests that the overall pattern of stimulation, not simply the retinal locus of motion, must be considered.

Third, both object and self-motion generally take place within a stationary environmental frame of reference. $\mathrm{Ob}$ ject motion is specified by the displacement of a surface relative to more distant "background" surfaces of the en- 
vironmental surround, and self-motion by flow of the environmental surround itself (Gibson, 1968). The order of surfaces in depth may be given by texture occlusion and disocclusion, motion perspective, stereopsis, and so on. Consistent with this notion is the evidence that both vection and postural adjustments are driven by motion of surfaces perceived to be in the background, but not by motion of surfaces perceived to be in the foreground (Brandt, Wist, \& Dichgans, 1975; Ohmi, Howard, \& Landolt, 1987).

Finally, the perception of self-motion could be restricted to optical accelerations or temporal frequencies correlated with the dynamics of normal postural sway, and higher rates of optical flow attributed to object motion (Stoffregen \& Riccio, 1990). Thus Berthoz, Lacour, Soechting, and Vidal (1979) reported that the frequency responses of vection and postural adjustments are both dominated by the low frequency range, below $0.2 \mathrm{~Hz}$.

Let us now review the evidence on peripheral dominance.

\section{Vection}

The classic example of vection is the "train illusion," in which an observer seated in a stationary train feels as if he or she is moving when a train on the adjacent track pulls out. Initial research suggested that peripheral stimulation was necessary to induce such a sensation of selfmotion. In the case of circular vection, or perceived yaw about the vertical axis in a rotating drum, Brandt et al. (1973) reported that circular displays $30^{\circ}$ or $60^{\circ}$ in diameter presented from $45^{\circ}$ to $75^{\circ}$ in the periphery were sufficient to evoke vection with a subjective intensity and velocity close to that obtained with full-field stimulation. On the other hand, a central $60^{\circ}$ region had a reduced effect, and a central $30^{\circ}$ region had no effect at all. Similar results were subsequently obtained for roll vection, or rotation about the line of sight (Held, Dichgans, \& Bauer, 1975), and for linear vection, including translation along the anterior-posterior (A-P) axis (Berthoz, Pavard, \& Young, 1975) and the vertical axis (Johansson, 1977). It quickly came to be accepted that the retinal periphery dominated in the perception of self-motion.

However, more recent results call this conclusion into question (G. J. Andersen, 1986). G. J. Andersen and Braunstein (1985), who used radial flow patterns that simulated movement through a three-dimensional cloud of dots, reported linear vection with central stimulation as small as $7.5^{\circ}$. In a direct replication of Brandt et al. (1973), Post (1988) studied circular vection with a $30^{\circ}$ display and reported no effect of eccentricity on the intensity and velocity of vection. Both central and peripheral effects were $60 \%$ of that obtained with a full-field display, due to the reduced area of stimulation.

In a critical experiment, Howard and Heckmann (1989) manipulated central and peripheral information for foreground and background surfaces in a rotating drum, and obtained circular vection with central displays as small as $13.5^{\circ}$. The results showed no difference between central and peripheral stimulation when the displays were equated for area and presented in the background, both yielding about $50 \%$ of full-field intensity. ${ }^{1}$ They concluded that vection is primarily controlled by two factors that trade off: retinal area of stimulation and motion of the perceived background surface. Similar results were found by Delorme and Martin (1986) for linear vection in an oscillating room, showing persistent vection with both $40^{\circ}$ central stimulation and full peripheral stimulation (central $80^{\circ}$ masked) as long as the moving surface was in the background; no vection was obtained for moving foreground surfaces with a stationary background. Frost and his colleagues (Shaver, Telford, \& Frost, 1991; Telford \& Frost, 1991) have also reported central vection with a moving background surface when the order in depth was specified by either texture occlusion and disocclusion or stereopsis.

The previous discrepancies can be explained by these two factors, for in most of the earlier studies, either retinal area was not equated or central stimulation was presented in the foreground (see Howard \& Heckmann, 1989). They also account for the "airplane" version of the train illusion, in which vection is induced by a moving background surface viewed centrally through a small window. In sum, it is now clear that vection can be induced with small areas of central stimulation, contrary to the predictions of the peripheral dominance hypothesis.

\section{Postural Adjustments}

Research on postural adjustments induced by optical flow followed a similar development, often influenced by the findings on vection. Initially, investigators reported that spontaneous standing sway increased with the occlusion of peripheral vision but not with the occlusion of central vision, suggesting that the periphery played the dominant role (Amblard \& Carblanc, 1980; Begbie, 1966; Dickinson, 1969; Dickinson \& Leonard, 1967). When postural adjustments were actively driven by optical flow displays, Lestienne, Soechting, and Berthoz (1977) found that the magnitude of A-P body tilt dropped as the location of a vertical strip of moving texture shifted from the periphery into $20^{\circ}$ central vision. However, these studies suffered from a number of methodological problemsnotably, a failure to equate the area of central and peripheral stimulation.

In contrast, more recent experiments have yielded significant postural effects with central stimulation. Paulus, Straube, and Brandt (1984) found no difference in spontaneous body sway with $30^{\circ}$ central vision and full peripheral vision (central $30^{\circ}$ masked). Moreover, when equated for area, $30^{\circ}$ of central vision actually yielded substantially less sway than did $30^{\circ}$ of peripheral vision, suggesting a greater central sensitivity for postural control. McCarty and Ashmead (1991) found a similar central advantage with both stationary and moving LED arrays. In a similar vein, Delorme and Martin (1986) reported that A-P sway was induced in a swinging room both with $40^{\circ}$ central vision and with peripheral vision (central $80^{\circ}$ masked), but only when the moving surface was in the background. De Graaf, van Asten, and Gielen (1990) found comparable results for lateral sway driven 
by roll stimulation $\left(80^{\circ}\right.$ "windmill" patterns oscillating about the line of sight), both with peripheral vision (central $50^{\circ}$ masked) and with $\geq 35^{\circ}$ central vision. No effects were found with $\leq 25^{\circ}$ central stimulation, but the masks were computer-generated and did not specify a background surface.

This evidence demonstrates that postural adjustments can be obtained with central vision, contrary to the predictions of the peripheral dominance hypothesis. However, there are indications of a more subtle type of differential sensitivity, such that retinal locus interacts with the structure of the optical flow pattern. Stoffregen (1985, 1986) found that $60^{\circ}$ displays of lamellar (parallel) flow induced body sway in both central and peripheral vision, whereas radial flow patterns had an effect in central vision but not at an eccentricity of $90^{\circ}$. This led him to propose that the peripheral retina is specialized to detect lamellar flow for postural control, whereas the central retina can detect both lamellar and radial flow. G. J. Andersen and Dyre (1989) confirmed postural effects of both radial and lamellar flow in $15^{\circ}$ central vision.

A similar interaction was reported for rotary flow by de Graaf et al. (1990), who used $35^{\circ}$ and $50^{\circ}$ windmill patterns. When the center of rotation was at the fixation point, the correlation between body sway and display oscillation was high, but when the center of rotation was $25^{\circ}$ in the periphery, it dropped dramatically. Thus, central vision appears to extract rotary flow for postural control, whereas peripheral vision does not.

In sum, there is consistent evidence that postural adjustments, like vection, can be induced in both central and peripheral vision. Both phenomena are influenced by the area of stimulation and motion of a background surface, results that are consistent with ecological conditions of selfmotion. Significantly, the central retina appears to extract radial, rotary, and lamellar flow patterns for postural control and vection, whereas the peripheral retina appears to extract only lamellar flow for these tasks.

\section{Perception of Heading}

In the research reported here, the role of peripheral information was investigated in the third domain, that of perceiving the direction of self-motion. Given the evidence just reviewed, it is doubtful that the peripheral dominance hypothesis is likely to hold for heading any more than it does for vection and postural control. Although it is worth it to properly test the strong version of the hypothesis in this domain as well, it is perhaps more important to determine the role that different retinal regions play in extracting radial and lamellar flow patterns for the perception of heading.

What might lead us to expect differential retinal sensitivity to information for self-motion? In previous studies, we have found translational heading judgments to be highly accurate (on the order of $1^{\circ}$ of visual angle) with low-density random-dot displays, with "velocity field" displays having a two-frame dot lifetime, and with velocity fields containing local directional and speed noise
(Warren, Blackwell, Kurtz, Hatsopoulos, \& Kalish, 1991; Warren, Morris, \& Kalish, 1988). These results led us to conclude that the visual system relies on the radial structure of the flow pattern to perceive translational heading, rather than the local focus of outflow. This is at least compatible with the peripheral dominance hypothesis, for the radial pattern could in principle be detected over a region of the periphery, and local dot motions could be "triangulated" to locate the heading point. In fact, the eccentricity scaling of optimal velocity sensitivity in the periphery (van de Grind, van Doorn, \& Koenderink, 1983) closely approximates the distribution of retinal velocities produced by walking over a ground surface while one looks straight ahead.

As pointed out by Koenderink and van Doorn (1987), as a constant-size patch of the optical flow field is sampled farther from the focus of outflow, the flow becomes increasingly lamellar and triangulation consequently less precise. Analyzed in the image plane, the angle $2 \alpha$ between two vectors separated by distance $2 r$ decreases with their distance $x$ from the focus of outflow:

$$
\alpha=\tan ^{-1}(r / x) \text {. }
$$

Given some error $\epsilon$ in determining the direction of local motions, the heading error $\delta$ of their intersection point would thus increase with more lamellar flow:

$$
(x+\delta)=r / \tan (\alpha+\epsilon) .
$$

As predicted, Crowell, Royden, Banks, Swenson, and Sekuler (1990) found a significant rise in discrimination thresholds for heading as a centrally presented patch of flow was sampled farther from the focus, when observers judged whether the second of two successive flow fields was to the right or to the left of the first. Assuming that $\epsilon$ is normally distributed, there should also be an asymmetry in heading error $\delta$, with perceived heading biased toward the far side of the actual heading point away from the fixation point, although this could not be determined with their relative heading task. This might lead one to expect an increased heading error with peripheral stimulation, if it is far from the focus of outflow.

With an annular sample of flow surrounding the focus of expansion, analogous to peripheral vision, one would not get such a systematic bias but might expect a decrease in heading precision, because error in local motion direction would produce a larger scatter of intersection points as one sampled farther from the focus. However, Warren et al. (1991; Hatsopoulos \& Warren, 1991) found no decline in performance with full-screen displays in which the directions of individual vectors were randomized within a $45^{\circ}$ envelope. This indicates that the redundancy in the global flow field allows the visual system to achieve high precision despite such local errors. Thus, on this score, a peripheral annulus of flow should allow accurate perception of heading.

However, there is another reason to expect that heading accuracy might decline with peripheral stimulation. A number of visual measures such as the minimum angle 
of resolution (MAR, the inverse of acuity), motion detection thresholds, optimum velocity sensitivity, and relative speed thresholds all jncrease linearly with eccentricity $\theta$ according to the function

$$
s=m \theta+1,
$$

where $s$ is a scaling factor by which to multiply the foveal value to obtain a corresponding value for any eccentricity (Levi, Klein, \& Aitsebaomo, 1984; McKee \& Nakayama, 1984; Sakitt \& Barlow, 1982; van de Grind, Koenderinck, \& van Doorn, 1986; van de Grind et al., 1983). This function can usually be related to the eccentricity scaling of neural properties such as receptive field size or velocity tuning. We might expect sensitivity to higher order pattern motion to obey the same scaling relation. However, Crowell et al. (1990) found no decline in their relative heading task when a fixed patch of the flow field was moved into the periphery, whether the visual angle of the patch was MAR scaled or held constant at $7^{\circ} \times 14^{\circ}$.

In the present study, we tested heading judgments while manipulating the retinal locus of stimulation. In the first experiment, we compared central and peripheral stimulation when the focus of outflow was in the central region. In the second and third experiments, we examined peripheral sensitivity to radial flow by varying the eccentricity of the focus of outflow. The results contradict the peripheral dominance hypothesis and indicate a greater central sensitivity to radial flow patterns.

\section{EXPERIMENT 1 \\ Central Versus Peripheral Stimulation}

The purpose of the first experiment was to compare heading accuracy with central and peripheral stimulation. Radial flow patterns simulating observer translation through a three-dimensional cloud were created (see Figure 1), in which only a central circular portion was visible (central condition), only the peripheral annulus outside the circle was visible (peripheral condition), or the whole $40^{\circ}$ screen was visible (combined condition). The diameter of the inner circle varied from $10^{\circ}$ to $25^{\circ}$, with a fixation cross at its center. The heading direction varied horizontally within the central $8^{\circ}$, so the complete circumference of the radial pattern was visible in all conditions. The resulting retinal flow pattern was comparable to that obtained when one looks in the approximate direction of self-motion.

Heading information was equated across conditions by holding the number of dots constant, so that the number of vectors with which to make a "triangulation" estimate of the heading point was the same in all conditions. However, there were several remaining differences between central and peripheral displays. First, dot density was consequently higher in central than in peripheral conditions, so we ran a control condition to equate density. Second, dot size and central/peripheral area were not eccentricity scaled in all conditions. However, the $15^{\circ}$ and $20^{\circ}$ conditions did provide appropriate MAR scaling of central
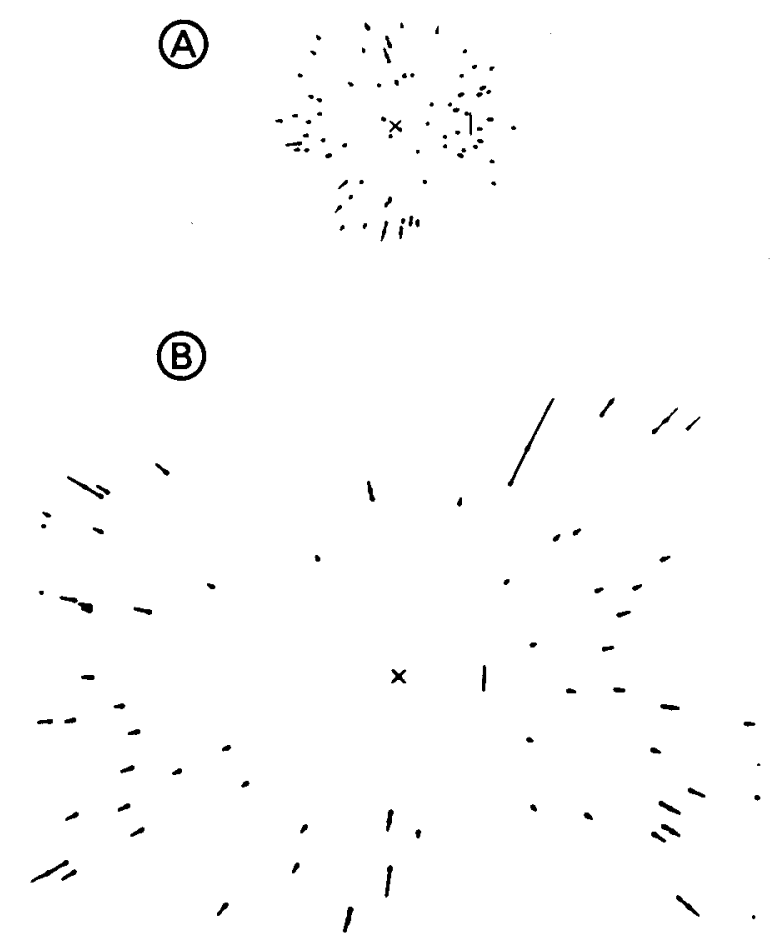

Figure 1. Velocity field for displays in Experiment 1. (A) Central condition. (B) Peripheral condition. (Mask diameter $=15^{\circ}$; heading direction, $1^{\circ}$ to right of fixation cross; heading angle, $4^{\circ}$ to left of target line.)

and peripheral area, and we ran a control condition to make sure that peripheral dot motion was above threshold in all conditions. Thus, we attempted to control the information in the central, peripheral, and combined conditions so that any differences in performance could be ascribed to differential retinal sensitivities.

\section{Method}

Observers. Eight graduate students and staff at Brown University were paid to participate. Two of these observers were also run in the control conditions. All had normal or corrected-to-normal vision and, with the exception of the first author, were participating in an optical flow experiment for the first time and were uninformed about the purposes of this study.

Displays. Displays depicting self-motion through a threedimensional cloud of dots were generated on a Raster Technologies Model One/380 graphics terminal hosted by a MicroVaxIl computer. They were presented on a Sony GDM-1901 monitor with a $60-\mathrm{Hz}$ refresh rate, a medium-short $\mathrm{P} 22$ phosphor, and a pixel resolution of $1,280 \times 1,024$, subtending $40^{\circ} \times 32^{\circ}$ (horizontal $\times$ vertical). The screen was viewed binocularly at the projectively correct distance of $45 \mathrm{~cm}$, using a black viewing box with a rectangular window at one end and a chinrest at the other. There is little agreement in the literature on what is meant by "central" and "peripheral" vision. Our reading suggests that displays up to $20^{\circ}$ in diameter are generally considered to be in central vision, whereas larger displays are said to include peripheral vision. We thus believe our $40^{\circ}$ display stimulated a portion of what is typically called peripheral vision.

Eyeheight units (e) were used as a distance metric, one standing eyeheight being approximately $1.6 \mathrm{~m}$. The dots were randomly posi- 
tioned in a cubic volume with its near surface at a distance of $1 \mathrm{e}$ $(1.6 \mathrm{~m})$, its far surface at a distance of $20 \mathrm{e}(32 \mathrm{~m})$, and its sides off screen. Dots were single white pixels $1.9^{\prime}$ of arc in diameter with a luminance of $118 \mathrm{~cd} / \mathrm{m}^{2}$ on a black background of $0.2 \mathrm{~cd} / \mathrm{m}^{2}$, and did not expand with motion. A display consisted of eight images that were cycled 10 times at 22 frames $/ \mathrm{sec}$, with an SOA of $45 \mathrm{msec}$. Each individual dot had a lifetime of 4 frames and was then replaced with another dot at a random position in the field, and dot lives were interlaced such that one fourth of the dots were replaced on each frame. This type of display allowed us to compute an occluding mask with the capabilities of the graphics system. The simulated observer speed was $11 \mathrm{e} / \mathrm{sec}(17.4 \mathrm{~m} / \mathrm{sec})$, yielding optical velocities ranging between approximately $0 \% \mathrm{sec}$ and $42^{\circ} / \mathrm{sec}$ and interframe displacements of $0^{\circ}$ to $2^{\circ}$, depending on a dot's depth and distance from the focus of outflow.

On each trial, a fixation " $X$ " with a diameter of $1^{\circ}$ appeared at the center of the screen for $1 \mathrm{sec}$, followed by dot motion for $3.6 \mathrm{sec}$. The simulated heading direction varied randomly between $0^{\circ}, \pm 1.0^{\circ}, \pm 2.0^{\circ}, \pm 3.0^{\circ}$, and $\pm 4.0^{\circ}$ to the left $(-)$ or right $(+)$ of the center of the screen. In the last frame of the display, a vertical $1.5^{\circ}$ target line appeared and remained visible together with the last frame of dots until the observer responded. To determine observer accuracy, the heading angle between the heading direction and the target varied randomly between $\pm 0.5^{\circ}, \pm 1.0^{\circ}, \pm 2.0^{\circ}$, and $\pm 4.0^{\circ}$. Observers were instructed to push one of two buttons to indicate whether it looked as if they would pass to the left (-) or right (+) of the target if they continued on their current path. Chance performance was thus $50 \%$ correct.

In the central condition, only dots within a circular border centered at the fixation point were visible, the rest being occluded by a computer-generated black peripheral mask. In the peripheral condition, only dots outside the circle were visible, with a black central mask. In the combined condition, dots were visible on the full screen. The diameter of the circular border varied between $10^{\circ}$, $15^{\circ}, 20^{\circ}$, and $25^{\circ}$, within the rectangular $40^{\circ} \times 32^{\circ}$ screen. In the peripheral condition, this left an annulus of dots with a horizontal thickness varying from $15^{\circ}$ to $7.5^{\circ}$ and a vertical thickness varying from $11^{\circ}$ to $3.5^{\circ}$. The areas of central and peripheral flow were, respectively, 79 and $1,121 \mathrm{deg}^{2}$ with the $10^{\circ}$ mask, 177 and $1,023 \mathrm{deg}^{2}$ with the $15^{\circ}$ mask, 314 and $886 \mathrm{deg}^{2}$ with the $20^{\circ}$ mask, and 491 and $709 \mathrm{deg}^{2}$ with the $25^{\circ}$ mask. Dots were occluded (or disoccluded) as they crossed the border of the mask.

To equate heading information (number of "triangulation" vectors) across conditions, the number of dots in each frame was held constant at approximately 90 dots. However, this meant that dot density was necessarily higher in the central than in the peripheral condition. We thus ran 2 observers in a density control condition, in which dot density was held constant at $0.16 \mathrm{dots} / \mathrm{deg}^{2}$ with the $20^{\circ}$ mask only, yielding approximately 50 dots in the central, 150 dots in the peripheral, and 200 dots in the combined conditions. They also received a $10^{\circ}$ central condition with just 20 dots, for a density of $0.25 \mathrm{dots} / \mathrm{deg}^{2}$, compared with $1.15 \mathrm{dots} / \mathrm{deg}^{2}$ in the 90-dot test condition.

Finally, because we did not MAR scale the sizes of individual dots, we also ran two observers in a translational motion control condition with peripheral displays, to ensure that this did not impair the peripheral detection of dot motion. Displays presented horizontal motion of a cloud of 90 dots at each of the four mask sizes, and the observer's task was to judge whether dot motion was leftward or rightward on the screen. These translational displays were matched with the heading displays by equating optical velocity at an eccentricity of $15^{\circ}$, so that a comparable range of velocities was present in the periphery.

Procedure. Trials were blocked by mask size in a counterbalanced order, and within that, blocked by condition in a randomly chosen order. Before each mask size block, observers received 15 practice trials with feedback at that mask size ( 5 in each condition), followed by 216 test trials without feedback ( 72 in each condition).
This made for a total of 864 test trials presented in two 90 -min sessions. Density control trials followed in a third session for 2 observers, with 216 trials in the $20^{\circ}$ condition and 72 trials in the $10^{\circ}$ central condition, and 192 translational motion control trials in a fourth session for 2 observers.

The data were collapsed across heading direction and positivenegative heading angles for analysis, yielding 18 trials at each unsigned heading angle. As in our previous studies, heading thresholds were calculated from these values by fitting each observer's data (percent correct by absolute heading angle) with an ogive, performing a $z$ transformation on percent correct, and computing a linear regression. The heading angle at which the regression line reached $75 \%$ correct was adopted as the threshold. All observers showed clear thresholds in each condition.

However, this method assumes that performance is symmetrical for positive and negative heading angles, and that there is no bias toward one side of the target or the other. In past studies of translational heading with free fixation, we have found no asymmetry, but with a central fixation point, we were concerned about a possible heading bias. To determine any constant heading error, we recoded heading angle as positive for "near" trials (actual heading on the near side of the target toward the fixation point) and negative for "far" trials (actual heading on the far side of the target away from the fixation point), plotted the percentage of "near" responses as a function of this near-far angle, and fit the data with an ogive as before. The point of subjective equality at which this curve crosses the $50 \%$ chance line is the near-far angle at which the observer reports heading straight toward the target, and taking its opposite sign gives the constant error. For example, if the observer reports heading straight at the target when the heading is actually $-1^{\circ}$ to the far side of the target, this corresponds to a $+1^{\circ}$ (near) heading error. The point at which the curve crosses the $75 \%$ line yields the difference limen, a measure of precision about the constant error value (see Kling \& Riggs, 1971, for details of this method).

\section{Results and Discussion}

The percentage of correct responses in each condition appears in Figure 2. Performance remained high in the central condition even with a very small area of stimulation, but declined in the peripheral condition with increasing mask size. A repeated measures analysis of variance (ANOVA) revealed a large effect of condition $[F(2,14)$ $=100.63, p<.001]$, accounting for $54 \%$ of the total sum of squares, and no overall effect of mask size $[F(3,21)$ $=2.47$, n.s.], but there was a significant interaction $[F(6,42)=3.00, p<.05]$, accounting for $6 \%$ of the sum of squares. A separate ANOVA on the peripheral condition alone confirmed a mask size effect $[F(3,21)=4.20$, $p<.05]$, accounting for $38 \%$ of the sum of squares. Post hoc Tukey tests on individual cell means within a column or a row (Cicchetti, 1972) showed no differences between the central and combined conditions, but significant differences between both of them and the peripheral condition at mask diameters of $15^{\circ}, 20^{\circ}$, and $25^{\circ}$ $(p<.01)$. Within the peripheral condition, there were significant differences between the $10^{\circ}$ and $20^{\circ}$ masks $(p<.05)$, and between the $10^{\circ}$ and $25^{\circ}$ masks $(p<.01)$.

In practical terms, this significant difference for the peripheral condition translates into a rather small decline in heading accuracy. The mean heading threshold was $0.5^{\circ}$ in the central condition and $0.6^{\circ}$ in the combined condition, and in the peripheral condition it rose from $0.8^{\circ}$ 


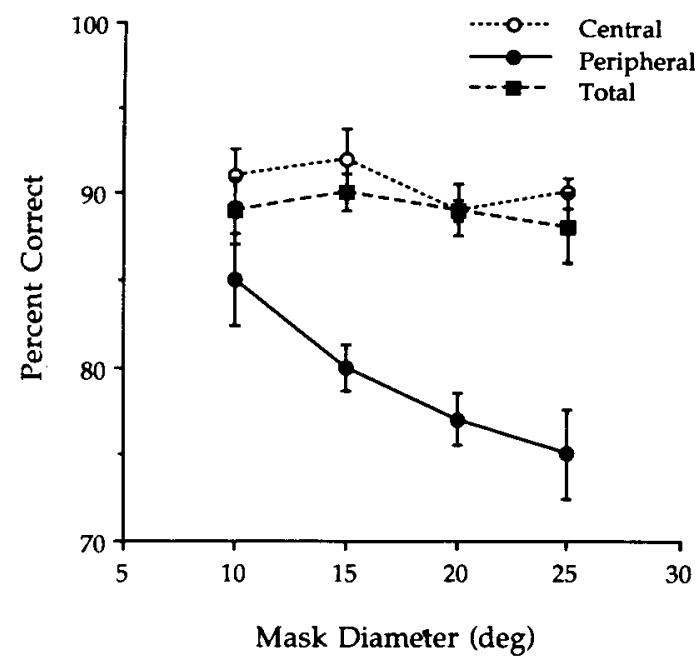

Figure 2. Mean percentage of correct responses as a function of mask size in the central, peripheral, and combined conditions (Experiment 1). Error bars indicate the standard error.

with a $10^{\circ}$ mask to $1.4^{\circ}$ with a $25^{\circ}$ mask. Thus, heading can be perceived quite well when central vision is obstructed. But contrary to predictions of the peripheral dominance hypothesis, heading accuracy is actually higher in central than in peripheral vision, and it declines with progressively larger central masks.

The error analysis suggested that the overall decline in peripheral performance is due both to a constant error and to a variable error (Figure 3). A small but significant constant "near" heading error of over $0.5^{\circ}$ toward the fixation point occurred in the $20^{\circ}$ and $25^{\circ}$ peripheral con- ditions, which is meaningful given that the focus of outflow only varied within $\pm 4^{\circ}$ from the fixation point. An ANOVA revealed a main effect of condition $[F(2,14)=$ $9.68, p<.002$ ], accounting for $16 \%$ of the total sum of squares, and no effect of mask size $[F(3,21)=0.97$, n.s. $]$, but there was a significant interaction $[F(6,42)=6.53$, $p<.001]$, accounting for $25 \%$ of the sum of squares. Tukey tests showed that the constant error was greater in the peripheral condition than in the central and combined conditions for $20^{\circ}$ and $25^{\circ}$ masks $(p<.05)$; within the central condition, the $25^{\circ}$ mask also had a significantly different error than did the $10^{\circ}$ and $15^{\circ}$ masks $(p<.05)$. Finally, the difference limen was higher in the peripheral than in the central and combined conditions as well, with a main effect of condition $[F(2,14)=55.57, p<$ .001 ], accounting for $30 \%$ of the total sum of squares, no effect of mask size $[F(3,21)=1.73$, n.s. $]$, and an interaction $[F(6,42)=3.14, p<.02]$, accounting for $13 \%$ of the sum of squares. Although these results are small and variable, we think that they suggest that the overall drop in performance was due to greater uncertainty about heading in the peripheral condition, which led to a slight bias toward the fixation point.

Before we can attribute these effects to differences in retinal sensitivity, several alternative explanations must be considered. First, it is possible that the difference between the central and peripheral conditions was due to higher dot densities in central displays. However, when we equated dot density in the $20^{\circ}$ density control condition, the difference remained: $91 \%$ correct in the combined condition, $92 \%$ correct in the central condition, but only $82 \%$ correct in the peripheral condition. Further, when the number of central dots was reduced from 90

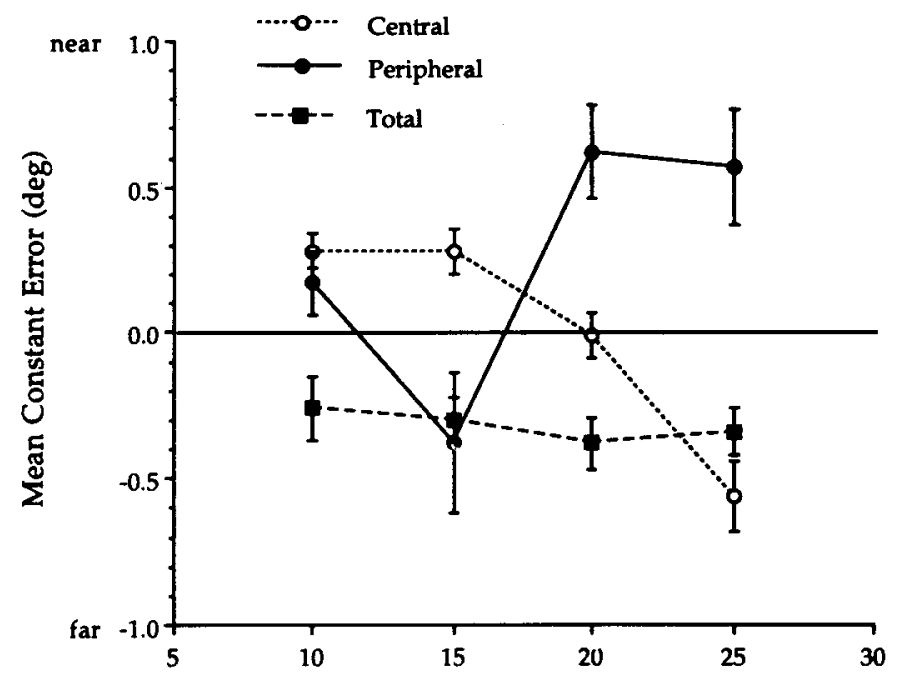

Mask Diameter (deg)

Figure 3. Mean constant heading error as a function of mask size (Experiment 1). A "near" error occurs when perceived heading is biased toward the fixation point. Error bars indicate the difference limen about the constant error value. 
to just 20 in the $10^{\circ}$ density control condition, performance remained at $95 \%$ correct, exactly where it was for these 2 observers with a high density. Thus, the centralperipheral difference cannot be attributed to differences in dot number or dot density.

Second, peripheral vision may have been at a disadvantage because the dots were not MAR scaled, making their motion more difficult to detect in the peripheral condition. However, performance in the translational motion control condition was $100 \%$ correct for both observers at all mask sizes. Thus, motion was well above threshold in all peripheral conditions, and the central-peripheral difference cannot be ascribed to a peripheral disadvantage in detecting dot motion per se. This also demonstrates that peripheral vision was not impaired by the use of shortlifetime dot motion.

Third, the central-peripheral difference as well as the decline in peripheral performance with mask size might be due to the retinal area of stimulation. To equate for this, central and peripheral display areas should be related by the scaling factor $s^{2}=(.33 \theta+1)^{2}$, where $\theta$ is one half the radius in the central condition or the eccentricity of the midpoint of the annular ring in the peripheral condition (McKee \& Nakayama, 1984). This criterion was met by the $15^{\circ}$ condition, in which peripheral area was six times the central area, as required, and roughly by the $20^{\circ}$ condition, where peripheral area was three times the central area, compared to the required five times. In both cases, performance was still significantly higher in the central than in the peripheral condition, so the difference between central and peripheral stimulation is not due to retinal area. We also interpret the decline in peripheral performance with increasing mask size to be due to a loss of information in central vision rather than a loss of area in peripheral vision, although we cannot rule out an area effect without using a larger display.

Fourth, as noted in the introduction, we doubt that the central-peripheral difference is due to increased triangulation error with vectors far from the focus of outflow. Because the complete circumference of the pattern was visible, there is no reason to expect that heading error would increase, given the redundancy in the flow pattern and the visual system's tolerance of directional noise (Warren et al., 1991).

Finally, it might be argued that the use of feedback could have influenced the results by training observers to respond to extraneous cues. Feedback was provided only during five practice trials for each condition to orient observers to unfarmiliar, minimal displays, and it is doubtful that they could have learned and tested a cue-based strategy in so few trials. Usually, such strategies depend on the presence of confounding cues whose validity degrades in such a way as to explain the pattern of performance, and our displays were carefully constructed to eliminate confounding cues; no observer mentioned such a strategy during debriefing. In addition, the observers in this experiment were naive, which helps to avoid cuebased responses (Braunstein \& Todd, 1990).

Two main results can be drawn from the present pattern of data. First, contrary to the predictions of the pe- ripheral dominance hypothesis, peripheral vision yields a heading accuracy that is actually somewhat worse than that obtained with central vision. Second, $10^{\circ}$ central vision provides heading judgments as accurate as those obtained with a full screen, indicating that radial flow patterns can be detected in central vision. The first result contrasts with that of Crowell et al. (1990), who found no decline in performance when a fixed patch of the flow field was moved into the periphery.

These results suggest that central vision may be somewhat more accurate than peripheral vision in determining heading. We examined this issue further in the next experiment, by testing how well locally radial flow could be detected in the periphery.

\section{EXPERIMENT 2 Eccentricity of the Focus of Outflow}

In Experiment 1, perception of heading was slightly more accurate with central than with peripheral vision. However, in those displays, the focus of outflow was always located in the central region, so that locally radial flow was present centrally but not peripherally. It is possible that peripheral vision would exhibit equal heading accuracy with radial flow centered in the periphery.

In Experiment 2, we compared central and peripheral sensitivity to radial flow by varying the eccentricity of the focus of outflow in full-screen displays. If peripheral vision can extract locally radial flow, performance should be unaffected by eccentricity. On the other hand, if there is peripheral insensitivity to radial pattern motion, performance should deteriorate with eccentricity.

\section{Method}

Observers. Six of the observers from Experiment 1 were paid to participate. All were thus experienced with this type of display.

Displays. The apparatus and viewing conditions were the same as before. Full-screen displays of a random-dot cloud were the same as those in Experiment 1, with the following exceptions. To discourage shifts in fixation, each display consisted of eight frames that were cycled twice at 22 frames/sec, for a display duration of $682 \mathrm{msec}$. Previous research had found no decrement in performance with short displays down to $300 \mathrm{msec}$ (Crowell et al., 1990; Warren, Blackwell, \& Kurtz, 1992). The simulated heading direction varied in eccentricity between $0^{\circ}, \pm 1^{\circ}, \pm 2^{\circ}, \pm 5^{\circ}, \pm 10^{\circ}$, and $\pm 15^{\circ}$ to the left $(-)$ or right $(+)$ of the fixation point at the center of the screen. As before, the heading angle between this direction and the target varied between $\pm 0.5^{\circ}, \pm 1.0^{\circ}, \pm 2.0^{\circ}$, and $\pm 4.0^{\circ}$, and observers pressed a button to indicate whether it looked as if they would pass to the left or the right of the target.

Procedure. Observers received 10 practice trials with feedback and then 192 test trials without feedback in a random order, in one 30 -min session. The data were collapsed across positive and negative eccentricities, and the constant heading error and difference limen were calculated as in Experiment 1. There were four trials at each near-far heading angle in each eccentricity condition.

\section{Results and Discussion}

The percentage of correct responses at each eccentricity appears in Figure 4. As the focus of outflow moves farther from the fixation point, performance drops steadily $[F(5,25)=13.06, p<.001]$, accounting for $72 \%$ of 
the total sum of squares, until at $15^{\circ}$ eccentricity observers were only $66 \%$ correct. This decline is accounted for by a bias toward the fixation point and an increase in variable error (Figure 5). The constant "near" error increased to $2.3^{\circ}$ at $15^{\circ}$ eccentricity $[F(5,25)=4.74$, $p<.005$ ], accounting for $49 \%$ of the total sum of squares. In 5 out of 6 observers, the difference limen about this constant error also increased from $0.04^{\circ}$ at $0^{\circ} \mathrm{ec}-$ centricity to $0.14^{\circ}$ at $15^{\circ}[F(5,20)=4.75, p<.005]$, accounting for $54 \%$ of the total sum of squares. We interpret these results to mean that the ability to localize the focus of radial outflow deteriorates with eccentricity and is compensated for by a bias toward the fixation point as in Experiment 1.

What might account for the increasing error with eccentricity? First, because the eccentricity of the target was correlated with that of the focus $(r=0.96)$, it could be an artifact of a declining ability to localize the target in the periphery. However, to explain our results, such a target localization error would have to be quite largeon the order of $2.3^{\circ}$ at $15^{\circ}$ eccentricity. Vernier acuity with two line segments or two dots also declines with eccentricity, but only from about $6^{\prime \prime}$ of arc at $0^{\circ}$ to $1^{\prime}$ of arc at $20^{\circ}$ eccentricity (Westheimer, 1982). We thus do not believe that our results can be explained by peripheral error in target localization.

Another possibility is that heading estimates are made by triangulation from central vision, which would lead to larger error as central flow becomes more lamellar. The predicted function for constant heading error is given in Equation 2, where $r$ is the radius of central vision and $\epsilon$ is error in local vector direction. Assuming that the error in vector direction is normally distributed with $\epsilon=1 S D$, we can calculate the asymmetrical distribution of head-

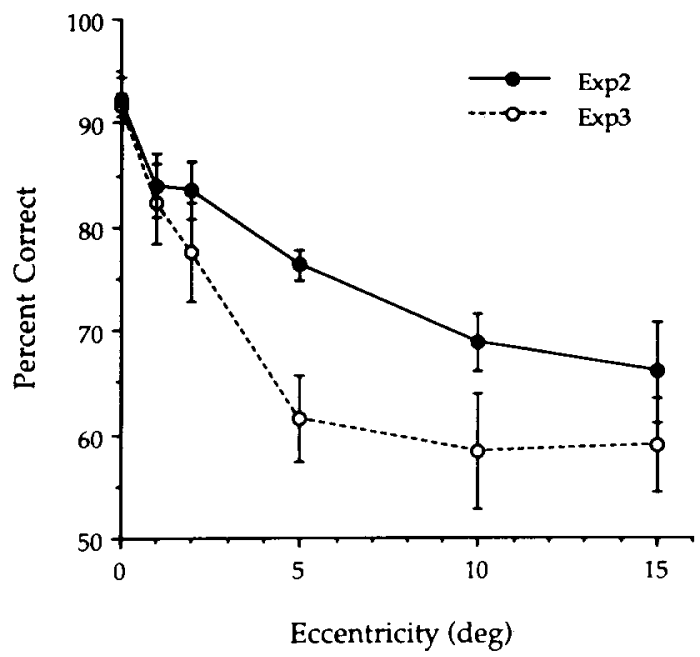

Figure 4. Mean percentage of correct responses as a function of the eccentricity of the focus of outhow with a three-dimensional cloud of dots (Experiment 2) and a ground plane of dots (Experiment 3). Error bars indicate the standard error.

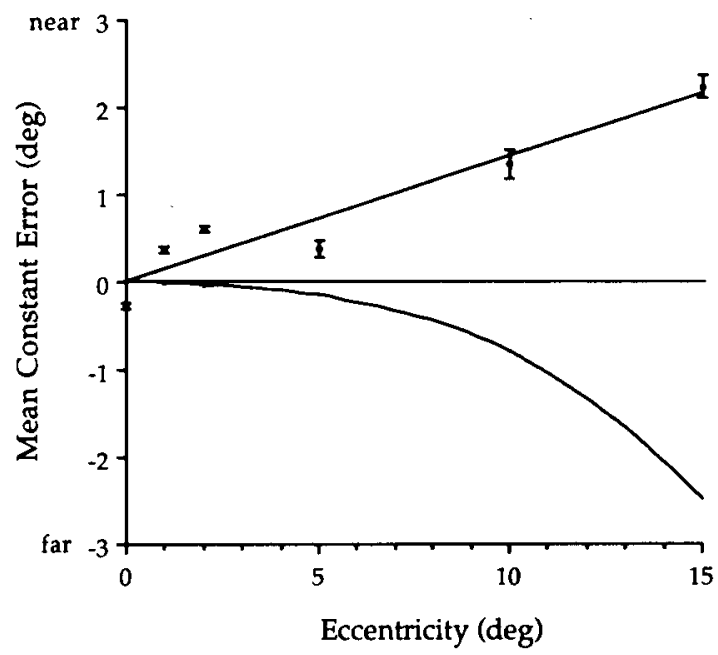

Figure 5. Mean constant heading error as a function of the eccentricity of the focus of outflow (Experiment 2). Upper curve is the linear regression best-fit line; lower curve is predicted from Equation 2 with $r=5^{\circ}, \epsilon=5^{\circ}$. Error bars indicate the difference limen about the constant error value.

ing errors at each eccentricity, with $\pm \delta= \pm 1 S D$. This model predicts, first, that heading errors should be skewed toward the far side of the heading point, biasing judgments toward the edge of the screen. In contrast, we found a center-screen bias, with constant errors on the near side of the heading point. Second, the shape of the theoretical curve provides a poor fit to the data. Figure 4 plots the predicted constant error (mean of signed $\delta$ ) as a function of eccentricity, with radius of central vision $r=5^{\circ}$ and error in vector direction $\epsilon=5^{\circ}$. Ignoring the sign of the constant error, these parameter values produced one of the best fits to our data, $r^{2}=.85$, but a simple linear regression accounted for more of the variance, $r^{2}=.91$ (slope $=0.144$, intercept $=-0.009$ ). Thus, we do not believe that our observers were estimating heading by triangulating from central vision.

A third possibility is that heading accuracy obeys the same eccentricity scaling as many other visual sensitivity measures. In the present experiment, constant error and variable error both increased approximately linearly with the eccentricity of the focus of outflow $\left(r^{2}=.81\right.$, slope $=0.009$, intercept $=0.039$, for the difference limen). We believe that the constant error is due to greater uncertainty about the location of heading due to lower sensitivity to radial flow in the periphery. Such a pattern of results might in principle be accounted for by eccentricity scaling of neural units sensitive to radial outflow patterns, and we will return to this possibility in the General Discussion.

We conclude that the heading system is most accurate with radial flow patterns that are centered on the fovea, and that peripheral vision beyond $5^{\circ}$ eccentricity is significantly less accurate. This is consistent with Stoffregen's $(1985,1986)$ finding that peripheral vision is less sensi- 
tive to locally radial flow for the task of postural control. We should note that it does not imply the somewhat paradoxical conclusion that observers must look in the direction of self-motion to perceive heading, for we have previously found thresholds of $1.5^{\circ}$ when tracking a moving fixation point at some distance from the heading direction, based on more complex retinal patterns than simple radial flow (Warren \& Hannon, 1990).

\section{EXPERIMENT 3 \\ Eccentricity of the Focus Under Locomotion Conditions}

Experiment 2 indicated that peripheral vision is less sensitive to radial flow patterns, using clouds of short-lifetime dots and a high observer speed. This finding was replicated in Experiment 3, under conditions that more closely approximated those for human locomotion, using a running speed and a ground plane with dots that persisted through the display.

\section{Method}

Observers. Six graduate students and staff at Brown University, with normal or corrected-to-normal vision, were paid to participate. All were experienced observers, having previously participated in other optical flow experiments, but only 1 (the first author) participated in the other experiments reported here.

Displays. The apparatus and viewing conditions were the same as before, but this time the displays depicted self-motion parallel to a random-dot ground surface at a running speed of $2.4 \mathrm{e} / \mathrm{sec}$ $(3.8 \mathrm{~m} / \mathrm{sec})$, similar to those used by Warren, Morris, and Kalish (1988). To prohibit shifts in fixation, each display consisted of only six frames that were presented once at 15 frames/sec, for a display duration of $333 \mathrm{msec}$. The ground plane extended to a pseudohorizon at a distance of $23.3 \mathrm{e}\left(37.3 \mathrm{~m}, 2.4^{\circ}\right.$ below the true horizon) and contained approximately 62 dots. The dots were single white pixels $\left(118 \mathrm{~cd} / \mathrm{m}^{2}\right)$ on a blue background $\left(90 \mathrm{~cd} / \mathrm{m}^{2}\right)$.

On each trial, the first frame of dots appeared for $1 \mathrm{sec}$ together with a fixation point at the center of the screen (a $0.5^{\circ}$ circle with a dot at the center), followed by dot motion; a vertical $1.5^{\circ}$ target line appeared in the last frame of dots, which remained visible until a response was made. As in Experiment 2, the simulated heading direction varied in eccentricity between $0^{\circ}, \pm 1^{\circ}, \pm 2^{\circ}, \pm 5^{\circ}, \pm 10^{\circ}$, and $\pm 15^{\circ}$ to the left $(-)$ or right $(+)$ of the fixation point, and the heading angle between this direction and the target varied between $\pm 0.5^{\circ}, \pm 1.0^{\circ}, \pm 2.0^{\circ}$, and $\pm 4.0^{\circ}$. Observers pressed a button to indicate whether it looked as if they would pass to the left or to the right of the target.

Procedure. Observers received 10 practice trials with feedback and then 192 test trials without feedback in a random order, in one 30-min session. The data were collapsed across positive and negative eccentricities.

\section{Results and Discussion}

The percentage of correct responses at each eccentricity appears in Figure 4. As eccentricity increases, performance drops sharply until it asymptotes at around 60\% correct with an eccentricity of $5^{\circ}[F(5,25)=15.04$, $p<.001]$, accounting for $75 \%$ of the total sum of squares.

These results agree with those of Experiment 2, as heading judgments deteriorate rapidly with eccentricity. An ANOVA comparing percentage correct for the two ex- periments yielded a main effect of experiment $[F(1,10)=$ $5.80, p<.05]$, but no experiment $\times$ eccentricity interaction $[F(5,50)=1.67$, n.s. $]$. The main effect could be due to several differences in the displays for Experiment 3, including an observer speed less than one fourth that in Experiment 2, a shorter display duration of $333 \mathrm{msec}$ compared with $682 \mathrm{msec}$, and only the bottom half of the radial flow pattern visible with a ground surface. Previous experiments on translational heading have shown no effects of display duration above $\mathbf{3 0 0} \mathbf{~ m s e c}$ (Crowell et al., 1990; Warren et al., 1992) and no differences in heading accuracy between ground surfaces and clouds (Warren et al., 1991), but a significant effect of speed (Warren et al., 1988). This suggests that the difference may be due to lower optical velocities in Experiment 3.

In sum, Experiments 2 and 3 both indicate that the heading system is most accurate with radial flow patterns that are centered on the fovea, whereas the periphery is less sensitive to locally radial flow.

\section{GENERAL DISCUSSION}

The present experiments extend recent findings on vection and postural control to the perception of heading. The results are consistent with previous work in which it has been shown that information about self-motion can be successfully extracted in central vision, and they indicate that central vision actually yields somewhat more accurate heading judgments than peripheral vision does. They are also consistent with Stoffregen's $(1985,1986)$ finding that radial flow elicits postural adjustments in central vision, but not in the periphery. Given the poor performance that we found at just $15^{\circ}$ eccentricity, this lack of response at $90^{\circ}$ is not surprising.

Summarizing the findings on perception of self-motion, it appears that central vision accurately extracts radial, rotary, and lamellar flow, whereas peripheral vision extracts lamellar flow but is less sensitive to radial and rotary flow. We can think of three possible explanations for this pattern of results. First and foremost, the peripheral dominance hypothesis is clearly inadequate. The literature repeatedly demonstrates that central stimulation is sufficient for perception of self-motion, and that central and peripheral stimulation yield comparable effects when they are equated for retinal area and specify a background surface. There is no retinally based "dominance" relationwe hope that the present results will put the final nail in the coffin of this enduring hypothesis.

A second explanation might be based on MAR scaling of simple velocity sensitivity, as shown by psychophysical studies of motion detection and relative speed thresholds. Greater sensitivity to radial and rotary flow in central than in peripheral vision would thus be ascribed to differential sensitivity to the local velocities that make up these flow patterns. However, the critical variable for distinguishing suprathreshold optical flow patterns is the direction of local velocities, and we know of no data on eccentricity scaling for direction discrimination. It is known 
that adding speed or directional noise to local dot motions does not greatly impair the visual system's ability to discriminate flow patterns (de Bruyn \& Orban, 1990; Warren et al., 1991; Watanamiuk, Sekuler, \& Williams, 1989). This is contrary to an explanation in terms of decreasing velocity sensitivity, because precise velocity measurements appear unnecessary.

A third possibility is that self-motion is determined on the basis of optical information rather than the retinal locus of stimulation (Gibson, 1968), but that central and peripheral vision are differentially sensitive to the type of information that is normally prevalent in that region. Such differential sensitivity could be due to eccentricity scaling of units selective for higher order patterns of motion. Let us consider this functional sensitivity hypothesis in more detail.

According to the hypothesis, central and peripheral vision are differentially sensitive to the information for object and self-motion that is characteristic of each retinal region. Consideration of the ecology of retinal flow during self-motion reveals that central flow can have a variety of patterns, whereas peripheral flow is nearly always locally lamellar. Described on a spherical projection surface, translation of the observer generates flow along longitudinal lines, so that it is locally radial near the axis of translation and lamellar near the "equator." Such patterns occur during locomotion and linear vection. In contrast, rotation of the observer generates flow along latitudinal lines, so that it is rotary near the axis of rotation and lamellar near the equator. These patterns occur during eye movements, head rotation, and circular and roll vection.

The consequences for central and peripheral vision have not been fully appreciated. If the observer looks in the direction of translation, central flow will be radial and peripheral flow will be lamellar. However, given the facts of optokinetic nystagmus and pursuit eye movements, if the observer looks anywhere else there will be an additional eye rotation. This yields retinal flow that is zero at the fovea, a complex central flow pattern that depends on environmental structure, and peripheral flow that is predominantly lamellar (Warren \& Hannon, 1990). In contrast, peripheral flow is radial only when the horizon off to the side is fixated during locomotion, and rotary only during head roll when a point off to the side that is rotating with the head is fixated; both are rare instances. Otherwise, peripheral flow is nearly always locally lamellar. Consequently, it might be expected that the visual system would be maximally sensitive to radial flow when the focus was centered on the fovea and that central vision would extract a variety of other flow patterns as well, but that the periphery would be less sensitive to radial and rotary flow.

One mechanism that could provide this differential sensitivity is eccentricity scaling of cortical units selective to pattern motion. Recent evidence for large-field units in area MST of the macaque that are selective for translation, expansion, contraction, rotation, and spiral mo- tion is suggestive in this regard ( $R$. Andersen, Graziano, \& Snowden, 1991; Saito et al., 1986; Tanaka \& Saito, 1989). The physiological data indicate a linear decrease in the number of expansion/contraction, rotation, and translation units with eccentricity, which could yield lower resolution for radial and rotary flow patterns in peripheral vision. In contrast, a lower number of translation units in the periphery should not be critical for extracting lamellar flow, because such flow is consistent over a larger retinal area. This architecture could account for our finding in Experiment 1 that performance declined with central masks, due to decreasing activation of large-field expansion units centered on the fovea. It could also explain our result in Experiments 2 and 3 that heading accuracy declined linearly with eccentricity, due to a decreasing density of such units in the periphery.

To these considerations we must add the problem of distinguishing self-motion from object motion. In contrast to self-motion, information that specifies object motion may appear anywhere on the retina. Indeed, Stoffregen and Riccio (1990) recently found that the timing of headdodging responses to an approaching object is highly accurate in both central vision and $90^{\circ}$ in the periphery. This could still be achieved with a lower density of expansionselective units in the periphery.

It is not yet clear what role MST units actually play in the analysis of object and self-motion. There is some evidence to suggest that expansion units are positioninvariant (Saito et al., 1986), which would make them ineffective for locating the center of outflow, although they may just be broadly tuned (Hatsopoulos \& Warren, 1991). The fact that expansion units are both size and speed selective (Tanaka \& Saito, 1989) would make them useful in extracting information for the time to contact of an approaching object, which is specified by the ratio of the object's visual angle to its rate of expansion (Lee, 1974). In either case, such motion mechanisms must distinguish radial flow due to self-motion from radial flow due to object motion, based on the area of stimulation and the order of surfaces in depth.

In sum, we suggest that the existing pattern of results can be accounted for by a functional sensitivity hypothesis. Self-motion is distinguished from object motion on the basis of optical information rather than the retinal locus of stimulation, but particular retinal regions appear more sensitive to the characteristic information. Further study of peripheral sensitivity to optical patterns specifying selfand object motion are necessary to test the hypothesis.

\section{REFERENCES}

Amblard, A. , CARblanc, B. (1980). Role of foveal and peripheral visual information iл the maintenance of postural equilibrium in man. Perceptual \& Motor Skills, 51, 903-912.

ANDERsen, G. J. (1986). Perception of self-motion: Psychophysical and computational approaches. Psychological Bulletin, 99, 52-65.

Andersen, G. J., Braunstein, M. L. (1985). Induced self-motion in central vision. Journal of Experimental Psychology: Human Perception \& Performance, 11, 122-132.

ANDERSEN, G. J., DYRE, B. P. (1989). Spatial orientation from optic 
flow in the central visual field. Perception \& Psychophysics, 45 , 453-458.

Andersen, R., Graziano, M., \& Sowden, R. (1991). Selectivity of area MST neurons for expansion/contraction and rotation motions. Investigative Ophthalmology \& Visual Science, 32, 823.

BEGBIE, G. H. (1966). The effects of alcohol and of varying amounts of visual information on a balancing test. Ergonomics, 9, 325-333.

Berthoz, A., Lacour, M., Soechting, J. F., Vidal, P. P. (1979). The role of vision in the control of posture during linear motion. Progress in Brain Research, 50, 197-209.

Berthoz, A., Pavard, B., Young, L. R. (1975). Perception of linear horizontal self-motion induced by peripheral vision (linear vection). Experimental Brain Research, 23, 471-489.

Brandt, T., Dichgans, J., Koenig, E. (1973). Differential effects of central versus peripheral vision on egocentric and exocentric motion perception. Experimental Brain Research, 16, 476-491.

Brandt, T., Wist, E. R., \&ichgans, J. (1975). Foreground and background in dynamic spatial orientation. Perception \& Psychophysics, 17, 497-503.

Braunstein, M. L., \& TodD, J.T. (1990). On the distinction between artifacts and information. Journal of Experimental Psychology: $\mathrm{Hu}$ man Perception \& Performance, 16, 211-216.

CicchetTI, D. V. (1972). Extension of multiple-range tests to interaction tables in the analysis of variance. Psychological Bulletin, 77. 405-408.

Crowell, J. A., Royden, C. S., Banks, M. S., Swenson, K. H., \& Sexuler, A. B. (1990). Optic flow and heading judgments. Investigative Ophthalmology \& Visual Science, 31, 522.

De BruYn, B., Orban, G.A. (1990). The role of direction information in the perception of geometric optic flow components. Perception \& Psychophysics, 47, 433-488.

De Graaf, J. B., van Asten, W. N. J. C., \& Gielen, C. C. A. M (1990). The effect of central and peripheral vision on postural balance of a human viewing a rotating scene. Unpublished manuscript.

Delorme, A., MarTin, C. (1986). Roles of retinal periphery and depth periphery in linear vection and visual control of standing in humans. Canadian Journal of Psychology, 40, 176-187.

Dichgans, J., \& BRANDT, T. (1978). Visual-vestibular interaction: Effects on self-motion perception and postural control. In R. Held, H. Leibowitz, \& H.-L. Teuber (Eds.), Handbook of sensory physiology: Vol. 8. Perception (pp. 755-804). New York: Springer-Verlag.

DiCKINSON, J. (1969). A note on the role of peripheral vision in static balancing. Ergonomics, $12,935$.

Dickinson, J., \& LEONARD, J. A. (1967). The role of peripheral vision in static balancing. Ergonomics, 10, 421-429.

Gibson, J. J. (1950). Perception of the visual world. Boston: Houghton Mifflin.

Gibson, J. J. (1954). The visual perception of objective motion and subjective movement. Psychological Review, 61, 304-314.

Gibson, J. J. (1968). What gives rise to the perception of motion? Psychological Review, 75, 335-346.

Hatsopoulos, N., WARREN, W. (1991). Visual navigation with a neural network. Neural Networks, 4, 303-317.

Held, R., Dichgans, J., \& Bauer, J. (1975). Characteristics of moving visual scenes influencing spatial orientation. Vision Research, 15, 357-365.

HowARD, I. P., HeCKmanN, T. (1989). Circular vection as a function of the relative sizes, distances, and positions of two competing visual displays. Perception, 18, 657-665.

Johansson, G. (1977). Studies on visual perception of locomotion. Perception, 6, 365-376.

KLING, J. W., RIGGS, L. A. (1971). Woodworth \& Schlosberg's Experimental psychology (3rd ed.). New York: Holt, Rinehart, \& Winston.

KoENDERINK, J. J., \& VAN DOORN, A. J. (1987). Facts on optic flow. Biological Cybernetics, 56, 247-254.

LEE, D. N. (1974). Visual information during locomotion. In R. B.
MacLeod \& H. Pick (Eds.), Perception: Essays in honor of James J. Gibson (pp. 250-267). Ithaca, NY: Cornell University Press.

LEE, D. N., * LISHMAN, J. R. (1975). Visual proprioceptive control of stance. Journal of Human Movement Studies, 1, 87-95.

Lestienne, F., Soechting, J., Berthoz, A. (1977). Postural readjustments induced by linear motion of visual scenes. Experimental Brain Research, 28, 363-384.

Levi, D. M., Klein, S. A., \& Artsebaomo, P. (1984). Detection and discrimination of the direction of motion in central and peripheral vision of normal and amblyopic observers. Vision Research, 24, 789-800.

MCCARTY, M. E., ASHMEAD, D. H. (1991). The efficacy of central visual stimulation for regulating postural sway. Unpublished manuscript.

McKeE, S. P., \& Nakayama, K. (1984). The detection of motion in the peripheral visual field. Vision Research, 24, 25-32.

Ohmi, M., Howard, I. P., LANDolt, J. P. (1987). Circular vection as a function of foreground-background relationships. Perception, 16, 17-22.

Paulus, W. M., Straube, A., Brandt, T. (1984). Visual stabilization of posture: Physiological stimulus characteristics and clinical aspects. Brain, 107, 1143-1163.

Post, R. B. (1988). Circular vection is independent of stimulus eccentricity. Perception, 17, 737-744.

SAKITT, B., \& BARLOW, H. B. (1982). A model for the economical encoding of the visual image in cerebral cortex. Biological Cybernetics, 43, 97-108.

Saito, H., Yukie, M., Tanaka, K., Hikosaka, K., Fukada, Y., IWAI, E. (1986). Integration of direction signals of image motion in the superior temporal sulcus of the Macaque monkey. Joumal of Neuroscience, 6, 145-157.

Shaver, S. W., Telford, L., Frost, B. J. (1991). The role of stereoscopic depth cues in centrally-mediated linear vection. Investigative Ophthalmology \& Visual Science, 32, 696.

StOFfregen, T. A. (1985). Flow structure versus retinal location in the optical control of stance. Joumal of Experimental Psychology: Human Perception \& Performance, 11, 554-565.

STOFFREGEN, T. A. (1986). The role of optical velocity in the control of stance. Perception \& Psychophysics, 39, 355-360.

Stoffregen, T. A., Riccio, G. E. (1990). Responses to optical looming in the retinal center and periphery. Ecological Psychology, 2, 251-274.

TANAKA, K., \& SAITO, H. (1989). Analysis of motion of the visual field by direction, expansion/contraction, and rotation cells clustered in the dorsal part of the medial superior temporal area of the Macaque monkey. Joumal of Neurophysiology, 62, 626-641.

Telford, L., \& Fost, B. J. (1991). The role of kinetic depth cues in centrally-mediated linear vection. Investigative Ophthalmology \& Visual Science, 32, 830.

VAN DE Grind, W. A., Koenderink, J. J., \& VAN DoOrN, A. J. (1986). The distribution of human motion detector properties in the monocular visual field. Vision Research, 26, 797-810.

VAn de Grind, W. A., van DoORn, A. J., Koenderink, J. J. (1983) Detection of coherent movement in peripherally viewed random-dot patterns. Joumal of the Optical Society of America, 73, 1674-1683.

WarRen, W. H., Blackwell, A. W., Kurtz, K. J. (1992). Minimal conditions for perception of heading from optical flow. (Manuscript in preparation)

Warren, W. H., BLACKWEll, A. W., Kurtz, K. J., Hatsopoulos, N. G., KALsh, M. L. (1991). On the sufficiency of the velocity field for perception of heading. Biological Cybernetics, 65, 311-320.

WARREN, W. H., \& HANNON, D. J. (1990). Eye movements and optical flow. Journal of the Optical Society of America A, 7, 160-169.

Warren, W. H., Morris, M. W., Kalish, M. (1988). Perception of translational heading from optical flow. Joumal of Experimental Psychology: Human Perception \& Performance, 14, 646-660.

Watanamiuk, S. N. J., Sekuler, R., Williams, D. W. (1989). Direction perception in complex dynamic displays: The integration of direction information. Vision Research, 29, 47-59. 
Westheimer, G. (1982). The spatial grain of the perifoveal visual field. Vision Research, 22, 157-162.

\section{NOTE}

1. There is still a slight asymmetry favoring peripheral motion: (1) Peripheral motion has an equivalent effect in the foreground and in the background, whereas central motion does not. (2) With simulta- neous central and peripheral motion in opposite directions, peripheral motion in the background dominates central motion in the foreground, but central motion in the background only cancels the effect of peripheral motion in the foreground.

(Manuscript received April 22, 1991;

revision accepted for publication December 13, 1991.) 